\title{
A bio-inspired computational language for kinesin nanomotor
}

\begin{abstract}
Kinesin nanomotor is a tiny vehicle that transports molecular cargoes within the cells. Many kinesin nanomotors can attach to a single cargo and coordinate their behaviors to transport the cargo. This behavioral coordination of kinesin nanomotors can be defined through a communicational language that kinesin nanomotors follow to transport the cargo. This paper proposes a computational language for kinesin nanomotor which is inspired by the nanomotor's natural behavior. In this technique, we have used behavioral Deterministic Finite Automaton (DFA) model of kinesin nanomotor which indicated internal intelligent and autonomous decision-making process of the nanomotor in response to its cell. In addition, the behavioral responses of kinesin nanomotor to its cell, behavioral DFA model of the nanomotor, were mapped to a computational regular language for the nanomotor. The proposed computational language for kinesin nanomotor was acceptable by the behavioral DFA model and also in good agreement with the natural behavior of the nanomotor. The development of such computational languages among intelligent and autonomous nanoparticles in nature paves the way for constructing potential bio-inspired nanorobotic systems as well as designing of some computational languages for their controlling.
\end{abstract}

Keyword: Kinesin nanomotor; Computational languages; Nanomotor; Deterministic Finite Automaton (DFA) model 